\title{
Capgras syndrome in dementia with Lewy bodies: a possible association of severe cortical Lewy body pathology
}

\author{
Shunsuke Koga ${ }^{1}$, Dennis W. Dickson ${ }^{1}$, Zbigniew K. Wszolek ${ }^{2}$ \\ ${ }^{1}$ Department of Neuroscience, Mayo Clinic, Jacksonville, Florida, United States \\ ${ }^{2}$ Department of Neurology, Mayo Clinic, Jacksonville, Florida, United States
}

Key words: Capgras syndrome, delusional misidentification syndrome, dementia with Lewy bodies, pathology

(Neurol Neurochir Pol 2021; 55 (6): 592-594)

\section{To the Editors}

Delusional misidentification syndrome is a group of delusional disorders in which a patient consistently believes that persons, places, objects or events have been altered [1]. Capgras syndrome is one of the presentations of delusional misidentification syndrome characterised by the recurrent and transient belief that a person, usually the spouse or a sibling, has been replaced by an imposter [2]. As with other forms of delusional misidentification syndrome, Capgras syndrome is associated with various disorders including psychiatric diseases (e.g. schizophrenia, schizoaffective disorder, depression), epilepsy, stroke, and neurodegenerative disorders such as Alzheimer's Disease (AD), Parkinson's Disease (PD), and dementia with Lewy bodies (DLB) [2]. DLB is pathologically categorised as Lewy body disease characterised by Lewy bodies and Lewy neurites composed of aggregated $\alpha$-synuclein in neurons [3]. Typical clinical features of DLB include dementia, fluctuating cognition, visual hallucinations, REM sleep behaviour disorder (RBD), and parkinsonism [3]. A subset of patients also develop Capgras syndrome [4], but any association between Capgras syndrome and $\alpha$-synuclein pathology remains unclear. In this letter, we describe the clinicopathological findings of a patient with DLB who developed Capgras syndrome.

The patient wasa 74-year-old Caucasian woman with 13-year history of formal education. She had no family history of parkinsonism or dementia. At the age of 63 years, she presented with a right foot tremor, cognitive decline with marked fluctuations, and RBD. One year later, she was diagnosed with PD and treated with pramipexole, without benefit. She developed unthreatening visual hallucinations, which were resolved after the discontinuation of pramipexole. She was started on carbidopa/levodopa, which improved her tremor. At the age of 67 years, she developed anxiety, depression, and Capgras syndrome. She was angry and irritable towards her husband and talked to him in the third person (as Mister). One year later, she was started on donepezil hydrochloride for her cognitive impairment with fluctuations, characterised by deficits in orientation, memory, and word-finding ability. She also manifested reduplicative paramnesia with several instances in which she thought her home was "not her real home" and more frequent manifestations of Capgras phenomena, specifically that her husband was not her real husband. She began treatment with quetiapine fumarate, but this did not improve the reduplicative phenomenon. On neurological examination, she had asymmetric rest tremor in her right hand, mild rigidity, and bradykinesia. Her postural stability was preserved, but her gait was slow and shuffling, with reduced arm swing.

Neuropsychological testing at the age of 67 years revealed a slow processing speed and impairments in divided attention, executive functions, and memory, consistent with mild cognitive impairment. Visuospatial function was preserved. She scored 135/144 on the Mattis Dementia Rating Scale. Since quetiapine fumarate was of no assistance, and pimavanserin had become available on the market, she was placed on this medication, but with an only minimal and transient improvement in her Capgras syndrome. Donepezil hydrochloride was discontinued, and she was treated with a combination therapy of rivastigmine and memantine hydrochloride, again without significant improvement. Her depression was treated with sertraline. None of these therapies were of any benefit for her Capgras syndrome.

Address for correspondence: Zbigniew K. Wszolek, M.D., Department of Neurology, Mayo Clinic Florida, 4500 San Pablo Road, Jacksonville, FL 32224,

USA; e-mail: wszolek.zbigniew@mayo.edu

Received: 4.10.2021 Accepted: 16.11.2021 Early publication date: 30.11 .2021

This article is available in open access under Creative Common Attribution-Non-Commercial-No Derivatives 4.0 International (CC BY-NC-ND 4.0) license, allowing to download articles and share them with others as long as they credit the authors and the publisher, but without permission to change them in any way or use them commercially. 

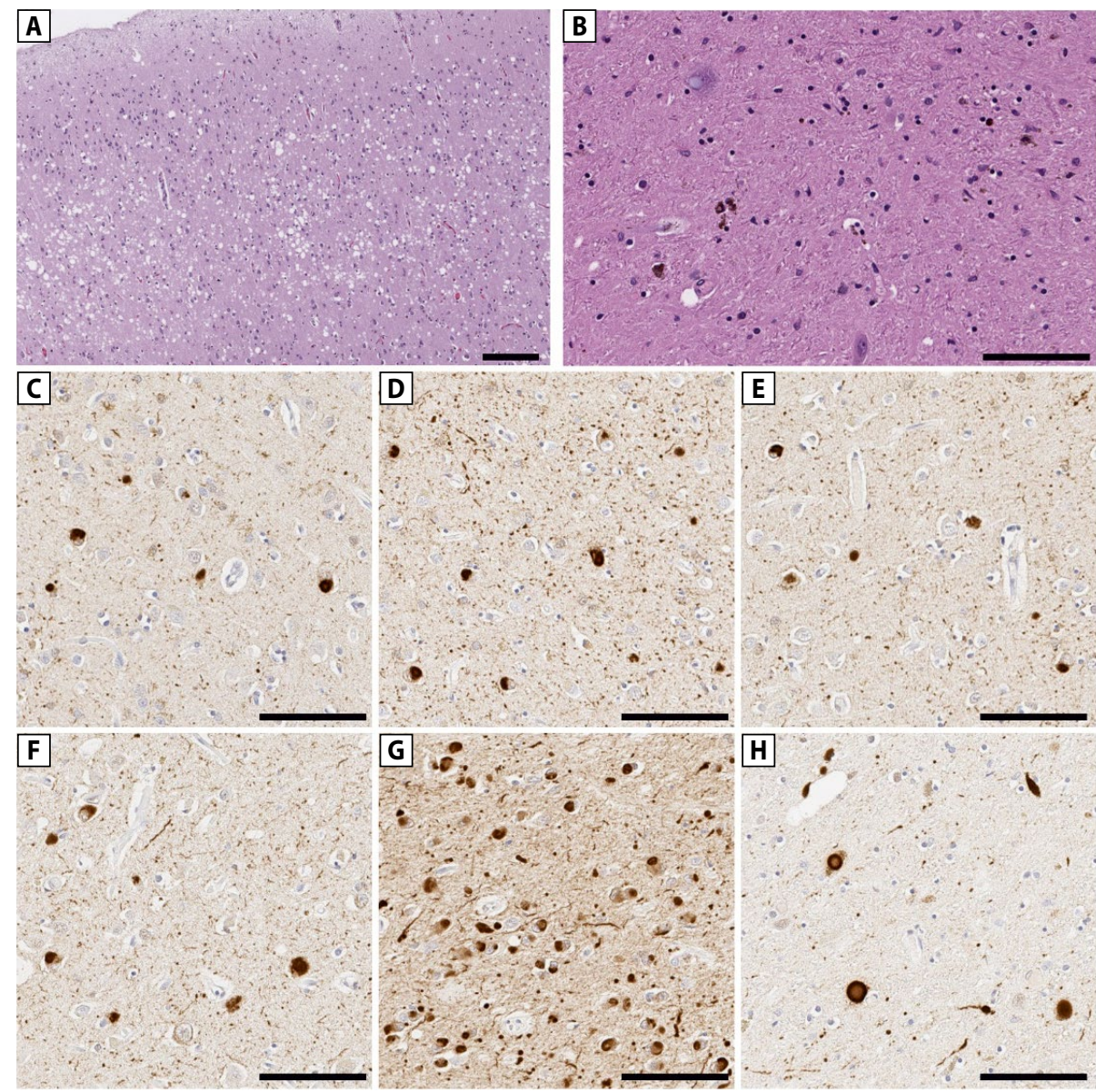

Figure 1. Histopathological findings. Haematoxylin and eosin (H\&E) stained sections (A-B) and immunohistochemistry for $\alpha$-synuclein (C-H). Spongiform change is visible on H\&E sections of entorhinal cortex (A). Substantia nigra has severe neuronal loss and gliosis with extracellular neuromelanin pigment (B). Abundant Lewy bodies and neurites are present in middle frontal gyrus (C), superior temporal gyrus (D), inferior parietal gyrus (E), cingulate gyrus $(\mathbf{F})$, amygdala (G), and substantia nigra (H). Scale bars: $200 \mu \mathrm{m}$ in $\mathbf{A} ; 100 \mu \mathrm{m}$ in B-H

Based on her dementia with fluctuations, RBD, and parkinsonism, she was diagnosed with probable DLB according to the consensus criteria [3]. She lived in a nursing home for the last four years of her life. Her Capgras features were later directed towards caregivers rather than her husband. She had significant fluctuations in motor and cognitive performance. On neurological examination at the age of 74 years ( 6 months prior to her demise), she was in a wheelchair and had a dystonic posture in her left hand and foot. She needed full assistance in all daily living functions.

A brain autopsy was performed after obtaining the consent of next of kin. The fixed left hemibrain weighed $450 \mathrm{~g}$. Macroscopic findings revealed mild cortical atrophy over the convexity. The frontal and temporal horns of the lateral ventricle were moderately dilated. The cortical grey mantle and cerebral white matter showed no unusual features. The hippocampal formation and amygdala had mild atrophy. The substantia nigra and the locus coeruleus had decreased pigmentation. The cerebellar sections showed no unusual features.

With thioflavin S fluorescent microscopy, many senile plaques and sparse neurofibrillary tangles (NFT) were detected in the neocortex. Most senile plaques were non-neuritic and diffuse amyloid deposits. Only a few of the senile plaques had dense amyloid cores. Only a few NFT were present in Sommer's sector, and a few senile plaques were present in the pyramidal layer and in the molecular layer of the dentate fascia. These findings were consistent with Braak NFT stage IV and Thal amyloid phase 3 . The neocortex had no significant neuronal loss and gliosis on haematoxylin and eosin stains, but there was a spongiform change in the temporal and limbic cortices (Fig. 1A). The hippocampus had normal neuronal populations. There was also spongiform change in the amygdala. The basal nucleus of Meynert had marked neuronal loss, with gliosis and vacuolation. The substantia nigra had a moderate-to-marked neuronal loss with extraneuronal neuromelanin and pigment-spheroid degeneration in the pars reticularis (Fig. 1B). Lewy bodies were frequent in residual neurons. The locus coeruleus had a moderate neuronal loss.

Immunohistochemistry for $\alpha$-synuclein (NACP, rabbit polyclonal, Mayo Clinic antibody) revealed abundant cortical Lewy bodies and Lewy neurites in the limbic and paralimbic 
cortices, as well as other cortices (Fig. 1C-F). Many Lewy neurites were detected in the $\mathrm{CA} 2 / 3$ region of the hippocampus. Marked Lewy-related pathology was also present in the endplate of the hippocampus and the subiculum, as well as the basal forebrain, hypothalamus, and amygdala (Fig. 1G). A moderate number of $\alpha$-synuclein-positive spheroids were present in the globus pallidus, and many dot-like and curvilinear Lewy neurites were present in the putamen. Lewy bodies and Lewy neurites were frequent in the substantia nigra (Fig. 1H), raphe nuclei, mesopontine tegmentum, locus coeruleus, dorsal motor nucleus of the vagus, glossopharyngeal nucleus, and medullary tegmentum. Based on these findings, neuropathological diagnoses of diffuse neocortical type Lewy body disease and early AD were made.

Capgras syndrome is not specific to Lewy body disease. The prevalence of Capgras syndrome in $\mathrm{AD}$ has been reported to be 6-13\% [5-7]. In a series of 47 patients with Capgras syndrome, Josephs found that 38 patients ( $81 \%$ ) had a neurodegenerative disease, most commonly Lewy body disease, followed by $\mathrm{AD}$ [4]. Josephs found that Capgras syndrome occurred a mean of eight years after the onset of parkinsonism, by which time all patients also had dementia and visual hallucinations. Our patient developed Capgras syndrome four years after the onset of parkinsonism, when she already had dementia with visual hallucinations.

These findings indicate that Capgras syndrome is probably associated with cortical involvement of Lewy body pathology. A possible association of dopamine-enhancing medications has been reported [8], but we did not find a clear correlation between the onset of Capgras syndrome and the dose of levodopa in our patient. In another study, Ferman et al. reported that the greater the Lewy body burden in the neocortex (particularly the frontal cortex), the earlier the onset of Capgras syndrome [9]. Given that our patient also had mild AD, this pathology may also relate to Capgras syndrome. Darby et al. investigated $17 \mathrm{pa}-$ tients with Capgras syndrome based on antemortem imaging studies and provided neuroanatomical correlates for impaired familiarity perception (left retrosplenial cortex) and belief evaluation (right frontal cortex) [10]. Lewy-related pathology and associated neurodegeneration in these brain regions may be associated with Capgras syndrome in Lewy body disease.

A limitation here is that only the left hemibrain was evaluated (the right half was frozen). We were not able to determine the role of right hemisphere pathology in her Capgras syndrome, although the right frontal cortex has been considered a significant region in Capgras syndrome $[1,10]$.

Further postmortem studies on Capgras syndrome are warranted to elucidate further clinicopathological correlations.

Acknowledgements: We would like to thank the patient and her family for their donation. We would also like to acknowledge Audrey Strongosky for her help of making the arrangements for the brain retrieval and shipment; Virginia Phillips, Jo A. Landino Garcia, and Ariston L. Librero (Mayo Clinic, Jacksonville) for histologic support; and Monica Castanedes-Casey (Mayo Clinic, Jacksonville) for immunohistochemistry support. This work was supported by the Albertson Parkinson's Research Foundation and the Rainwater Charitable Trust.

Disclosures: Dr. Koga receives research support by a Jaye F. and Betty F. Dyer Foundation Fellowship in progressive supranuclear palsy research and CurePSP. Dr. Dickson receives support from the National Institutes of Health grants (P50 NS072187) and the Rainwater Charitable Trust. Dr. Wszolek is partially supported by the Mayo Clinic Centre for Regenerative Medicine, the Mayo Clinic in Florida Focused Research Team Programme, and gifts from the Sol Goldman Charitable Trust, the Donald G. and Jodi P. Heeringa Family, the Haworth Family Professorship in Neurodegenerative Diseases Fund, and the Albertson Parkinson's Research Foundation. He serves as PI or Co-PI on Biohaven Pharmaceuticals, Inc. (BHV4157-206 and BHV3241-301), Neuraly, Inc. (NLY01-PD-1), and Vigil Neuroscience, Inc. (VGL101-01.001) grants. He serves as an external advisory board member for Vigil Neuroscience, Inc.

Conflict of interest: None.

\section{References}

1. Cipriani G, Vedovello M, Ulivi M, et al. Delusional misidentification syndromes and dementia: a border zone between neurology and psychiatry. Am J Alzheimers Dis Other Demen. 2013; 28(7): 671-678, doi: 10.1177/1533317513506103, indexed in Pubmed: 24164927.

2. Pandis C, Agrawal N, Poole N. Capgras' delusion: a systematic review of 255 published cases. Psychopathology. 2019; 52(3): 161-173, doi: 10.1159/000500474, indexed in Pubmed: 31326968.

3. McKeith IG, Boeve BF, Dickson DW, et al. Diagnosis and management of dementia with Lewy bodies: fourth consensus report of the DLB Consortium. Neurology. 2017; 89(1): 88-100, doi: 10.1212/ WNL.0000000000004058, indexed in Pubmed: 28592453.

4. Josephs KA. Capgras syndrome and its relationship to neurodegenerative disease. Arch Neurol. 2007; 64(12): 1762-1766, doi: 10.1001/ archneur.64.12.1762, indexed in Pubmed: 18071040.

5. Harwood DG, Barker WW, Ownby RL, et al. Prevalence and correlates of Capgras syndrome in Alzheimer's disease. Int J Geriatr Psychiatry. 1999; 14(6): 415-420, doi: 10.1002/(sici)1099-1166(199906)14:6<415::aid-gps929>3.0.co;2-3, indexed in Pubmed: 10398349.

6. Pereira GC, de Oliveira GC. Prevalence of capgras syndrome in alzheimer's patients: a systematic review and meta-analysis. Dement Neuropsychol. 2019; 13(4): 463-468, doi: 10.1590/198057642018dn13-040014, indexed in Pubmed: 31844501.

7. Harciarek M, Kertesz A. The prevalence of misidentification syndromes in neurodegenerative diseases. Alzheimer Dis Assoc Disord. 2008; 22(2): 163-169, doi: 10.1097/WAD.0b013e3181641341, indexed in Pubmed: 18525289.

8. Cannas A, Meloni M, Mascia MM, et al. Capgras syndrome in Parkinson's disease:twonewcasesandliteraturereview.NeurolSci.2017;38(2):225-231, doi: 10.1007/s10072-016-2765-9, indexed in Pubmed: 27848117.

9. Ferman TJ, Arvanitakis Z, Fujishiro H, et al. Pathology and temporal onset of visual hallucinations, misperceptions and family misidentification distinguishes dementia with Lewy bodies from Alzheimer's disease. Parkinsonism Relat Disord. 2013; 19(2): 227-231, doi: 10.1016/j.parkreldis.2012.10.013, indexed in Pubmed: 23182311.

10. Darby RR, Laganiere S, Pascual-Leone A, et al. Finding the imposter: brain connectivity of lesions causing delusional misidentifications. Brain. 2017; 140(2): 497-507, doi: 10.1093/brain/aww288, indexed in Pubmed: 28082298. 